\title{
From Prejudices and Marginalization to Social Integration: Some Examples from Italian Immigrant Literature
}

\author{
Dr. Nikica Mihaljević \\ Department of Italian Language and Literature, Faculty of Humanities and Social Sciences \\ University of Split Croatia \\ nikica@ffst.hr
}

Doi:10.5901/jesr.2014.v4n4p216

\begin{abstract}
In this paper the aim is to analyse, with an ecocritical reading, Salah Methnani and Mario Fortunato's "Immigrato" and Ingy Mubiayi's "Nascita". The novel "limmigrato" represents one of the first examples of the immigrant literature in Italy; it brings us the autobiographical story of a Tunisian who comes to Italy in search for better life conditions. In the society he finds, however, he will have to face prejudices and discrimination which will have as a consequence intolerance, rejection, and marginalization of immigrants. The story is presented as a journey from South to North of Italy, but actually it is a story of the research of identity in the new community. On the other hand, the short story "Nascita" is written by one of the authors whose partents have migrated to Italy and who has been raised in Italy, Ingy Mubiayi. The analysis shows differences of the two texts: in "Immigrato" the consequences of immigration are focalized, i.e. prejudices, racism, and marginalization, while in "Nascita" the attention is drawn to the immigrants who have integrated in the new society. In the analysis, the methodology of ecocriticism is used to discover the examples of discrimination and marginalization of some characters in the text, in order to evidence the so called "vertical society", which inevitably has as consequences discrimination, racism, and oppression of some of its members. Another aim of the analysis is to find the literary examples of how to overcome discrimination and make possible the building of more equitable society.
\end{abstract}

Parole chiave: ecocritica, letteratura d'immigrazione, società verticale, società orizzontale.

\section{Introduction}

Quando Serenella lovino espone e spiega i concetti base dell'ecologia letteraria, non si sofferma sull'analisi della letteratura di migrazione, anche se quel tipo di scrittura sembra offrire diversi esempi per promuovere e confermare i rapporti i quali sostiene proprio l'ecologia letteraria; l'ecologia letteraria mette in rilievo l'importanza della «cittadinanza ecologica», che "implica un modello democratico, che incoraggi la partecipazione dei cittadini ai progetti di conservazione ambientale, a cominciare da quelli relativi alla tutela e al recupero di spazi appartenenti alla comunità. Preservando e recuperando l'ambiente comune, è come se la società preservasse e recuperasse se stessa come struttura democratica." (Iovino 2006:55-56) L'ecologia letteraria rifiuta la visione dualistica del mondo, in quanto questa non può che causare un'impostazione piramidale della società, la quale, poi, a sua volta, porta verso la discriminazione ed emarginazione di alcuni suoi membri. In questo senso, "la cultura ambientale si presenta come un rinnovato orizzonte di valori. [...] Parlare di «strategia di sopravvivenza», allora, si fa meno metaforico; [...]." (Iovino 2006:59) II dualismo che caratterizza la società di oggi, invece, ce lo ricorda lovino

\begin{abstract}
[...] al posto di interdipendenze non riconosciute [...], stabilisce delle gerarchie e le «neutralizza». Tutto ciò non si limita però al mero piano concettuale; [...]. Questo conduce alla contrapposizione tra un polo "forte» e un polo "debole», nella quale si riconosce non solo l'antitesi di maschile e femminile, ma anche quella di colonizzatore e colonizzato, bianco e nero, etnie dominanti ed etnie dominate e via cosi. (lovino 2006:75)
\end{abstract}

Quindi, se affrontiamo un testo letterario con la metodologia dell'ecologia letteraria, assumiamo l'atteggiamento di chi individua e delinea la presenza dei dualismi in società e, di conseguenza, assume un atteggiamento critico nei confronti della società che non appoggia un discorso pacificatore e non cerca una soluzione per una cultura sostenibile, ma sostiene la gerarchia e le differenze. Tuttavia, la scelta di una cultura sostenibile ci si presenta, ricorda lovino, come "una strategia non casuale, ma consapevole, in grado di permettere all'umano di preservare se stesso e l'ambiente, e insieme di riconoscere nuovi valori e nuove categorie di pensiero." (Iovino 2006:59) La cultura ambientale, quindi, è finalizzata alla ridistribuzione nel mondo delle opportunità, in modo tale da raggiungere, alla fine, l'equità sociale tra tutti i suoi membri e, conseguentemente a ciò, la sostenibilità ecologica.

Riguardo alla specificità della letteratura di migrazione e la sua importanza per la società contemporanea, Paola 
Ellero ricorda che:

[...] le produzioni degli stranieri costituiscono, al di là del loro valore letterario, uno strumento per superare $i$ confini che ancora condizionano il nostro modo di pensare e vivere il fenomeno migratorio e la presenza di cittadini immigrati nel nostro Paese. Esse ci invitano a guardare la realtà, spesso viziata da stereotipi, attraverso gli occhi di chi ha cercato e trovato tra noi ospitalità, riuscendo anche a integrarsi. [...] La letteratura migrante in italiano può ricoprire un ruolo di rilievo in questo processo, perché riflette nel presente di questi nuovi vicini il nostro passato, non diverso sostanzialmente, anche se rimosso, di uomini e donne che hanno dovuto abbandonare la loro terra per cercare altrove una vita migliore. (Ellero 2010:4)

Infatti, uno dei primi romanzi di letteratura d'immigrazione in Italia, il romanzo di Salah Methnani e Mario Fortunato I' Immigrato, contiene una serie di esempi di pregiudizi sugli immigrati e, in quanto tale, si presta per una lettura ecocritica perché permette al lettore di notare la visione dualistica che caratterizza fortemente la società di oggi.

Per quanto riguarda l'inizio della letteratura migrante in Italia, Ellero rileva che si tratta della fine degli anni '80 e l'inizio degli anni '90 quando "I'Italia dal paese di emigrati si stava trasformando in terra d'immigrazione." (Ellero 2010:5) In un certo senso, secondo Ellero, fu il tentativo di regolarizzare l'immigrazione con la Legge Martelli che scatenò la scrittura immigrante:

Tra i primi a reagire a tale situazione fu lo scrittore marocchino Tahar Ben Jelloun, con Dove lo stato non c'è, e Salah Methnani, autore di Immigrato, uno marocchino, I'altro tunisino, di cultura francese, ma che decidono di scrivere sull'Italia in italiano. Queste opere vennero confezionate a quattro mani, ossia scritte insieme a uno scrittore o giornalista italiano che fece da coautore con la funzione di supporto linguistico. (Ellero 2010:5)

Questa prima fase di letteratura d'immigrazione viene denominata, ricorda Ellero, la «fase autobiografica», anche se gli elementi autobiografici non mancano nemmeno nelle opere successive di altri autori immigranti in Italia. Di conseguenza, nel presente articolo analizziamo, cercando di fornire una lettura ecocritica, un'opera di un autore di questa prima fase della letteratura di migrazione, e un'opera successiva, 0, più precisamente, un'opera del XXI secolo, di una scrittrice che appartiene alla csd. seconda fase di scrittura migrante, ovvero alla 'letteratura di seconda generazione' (Cfr. Ellero 2010:6), che si distingue per il fatto che gli autori-immigranti adoperano alcune modifiche nella scrittura dell'immigrazione:

\footnotetext{
Dopo la prima fase centrata sulle difficoltà della vita da migrante, si passa a una fase di creatività fortemente ancorata agli elementi culturali del paese di provenienza. Fondamentale rimane sempre il bisogno di comunicare, di aprire sempre una finestra sugli usi e costumi del paese di appartenenza, di valorizzare la propria cultura che effettivamente viene ignorata nel paese di accoglienza. (Ellero 2010:5)
}

Questi scrittori sono o nati in Italia o sono stati portati in Italia da piccoli e, quindi, sono cresciuti in Italia, accettando tutti i suoi costumi ed integrandosi completamente nella società nuova. A questi scrittori appartiene anche Ingy Mubiayi, "nata al Cairo da madre egiziana e padre zairese [...] [e] titolare di una libreria a Primavalle e nel 1977, appena arrivata nella capitale, andò ad abitare con la famiglia - una quindicina di persone tra genitori, fratelli, sorelle, fratellastri e sorellastre - a Casalotti." (Kuruvilla - Mubiayi - Scego - Wadia 2013:64) Analizzando, quindi, due opere di questi due scrittori di letteratura dell'immigrazione, uno della csd. prima fase, l'altra della seconda fase degli scrittori immigranti in Italia, uno uomo, l'altra donna, nel presente articolo si cerca di individuare, attraverso una lettura ecocritica, i cambiamenti introdotti nella scrittura dell'immigrazione rispetto alla sua fase iniziale, i quali rivelano anche la presenza degli esempi dei rapporti conflittuali degli immigrati con il paese d'origine.

\section{Immigirato; Aspettative, Pregiudizi, Disillusioni e Discriminazione}

Alfredo Luzi ci ricorda l'importanza della letteratura migrante, in quanto questa scrittura nasce dal bisogno di vincere l'isolamento nella società in cui si immigra:

La letteratura prodotta da migranti che, una volta giunti nel paese prescelto, sono spinti a scrivere per vincere il proprio isolamento, testimoniare in qualche modo la loro presenza, dialogare con il lettore italiano, esibire la propria diversità attraverso la scrittura, gioca un ruolo fondamentale nella costruzione di un immaginario collettivo relativo al paese d'accoglienza, a quello di provenienza, al confronto con i propri connazionali e con gli abitanti della nazione in cui sono approdati. (Luzi 2008:1)

Nell' Immigrato, che, secondo Luzi, "è un testo esemplare della configurazione di genere nella narrativa di 
migrazione e nello stesso tempo può essere assunto come modello di verifica dell'efficacia euristica del metodo imagologico" (Luzi 2008:1), scopriamo un racconto autobiografico, nel quale un ragazzo tunisino, colto e laureato in lingue, arriva in Italia nella ricerca di un lavoro e nella ricerca di quell'immagine del paese in cui il benessere dovrebbe essere a portata di mano di tutti. II paese che sceglie come paese in cui cercare lavoro gli si presenta come una scelta naturale, data la presenza della lingua italiana, attraverso i media, in Tunisia: "Fra le lingue straniere, non scelsi l'italiano, ma l'inglese e il russo. L'italiano, ne ero certo, l' avrei imparato comunque. Intanto, avevo già fatto i miei progressi: [...]. D'estate, ogni pomeriggio tranne il venerdí, mi sistemavo davanti al televisore per seguire i programmi della RAI. [...] Le trasmissioni non avevano i sottotitoli, ma a poco a poco le parole prendevano forma e senso nella mia testa." (Methnani Fortunato 2006:11) Paradossalmente, questa conoscenza dell'italiano, una volta che il protagonista si trova in Italia, gli complicherà la vita, perché conoscendo bene la lingua uscirà dall'immaginario collettivo in cui gli immigrati capiscono poco o per niente l'italiano, e questa sua caratteristica lo renderà ancora più indesiderato dagli italiani:

Da quando ho capito che la mia discreta conoscenza dell'italiano, invece di facilitare le cose, le complica, ho preso a parlare come ci si aspetta parli un «vu comprà». Negli ostelli e nelle mense dico: "Amigo incontrato stazione dire venire qua. Rubare me passaporto e soldi». Pare che questo linguaggio elementare tranquilizzi molto gli impiegati delle strutture per accoglienza degli immigrati. (Methnani - Fortunato 2006:58)

Il fatto che non conoscere l'italiano dalla parte degli immigrati tranquillizzi gli italiani ci conferma il loro bisogno di sentirli diversi, di percepirli differenti da se stessi, sia per la paura nei confronti di uno straniero, sia per poter giustificare gli intenti di emarginazione: per i residenti, il fatto che gli immigrati non conoscono la lingua, significa che non si sono integrati, e allora «è lecito» marginalizzarli.

Tuttavia, nonostante i buoni intenti, ben presto viene classificato come il solito immigrato clandestino che non ha intenzione di fare altro in Italia che rubare e perciò diventa vittima di numerosi pregiudizi e atteggiamenti discriminatori, senza, però, aver fatto nulla che costringeva altri personaggi intorno a lui a percepirlo in quel modo. Di conseguenza, in Italia si troverà a dover affrontare le condizioni di vita peggiori di quelle che ha lasciato in Tunisia e ad accettare qualsiasi tipo di lavoro per non dover rubare o prostituirsi come altri immigrati. Così, appena arrivato in Italia, si sente osservato come uno diverso, un intruso, il cui ogni atto, ogni singola mossa sembra essere ben nota a priori a tutti, nonostante si tratti soltanto delle illazioni legate ai pregiudizi: "Mi metto in fila per acquistare il pane e un po' di formaggio. [...] La signora che mi sta accanto, ogni tanto, mi guarda con la coda dell'occhio, poi stringe la borsa al petto. Faccio finta di niente." (Methnani - Fortunato 2006:15) Solamente per il colore della pelle del protagonista e per la sua fisionimia diversa, la donna si sente minacciata e fa un atto spontaneo di stringere la borsa per non permettergli di rubargliela. Nel romanzo gli episodi del genere saranno numerosi, quando soltanto la presenza di un uomo diverso, in quanto proveniente da un paese extracomunitario, basterà a spronare nei residenti la reazione ingiustificata di discriminazione. Inizia così il suo viaggio da una città italiana all'altra per trovare il posto migliore rispetto al precedente, ma ben presto essa si dimostrerà una ricerca inutile, perché più andrà verso nord troverà sempre più i casi del razzismo nonché l'accettazione, dalla parte dei residenti, degli stereotipi e dei pregiudizi che riguardano uno straniero. Si troverà, di conseguenza, di fronte al fatto di dover abbandonare quell'immagine dell'Italia che si era creato in infanzia, per lasciare spazio ad una nuova immagine, per niente positiva, dell'Italia che, se ti riduce ad essere la vittima di un «razzismo gentile», ti puoi considerare fortunato:

\begin{abstract}
Al Rivage, un circolo ricreativo, non si vede neanche l'ombra di un nordafricano. Mi sento addosso gli occhi di tutti. Faccio un sorriso di circostanza, vorrei bere una birra. Ma il proprietario dice: «Mi dispiace. Qui è proibito ai marocchini», solleva le spalle. Vorrei dire che non sono "un marocchino». Lascio perdere: tutto sommato, il suo è ancora un razzismo gentile. (Methnani - Fortunato 2006:23)
\end{abstract}

Gli esempi del razzismo nel romanzo ci aiutano ad individuare una visione dualistica che fortemente caratterizzava la società italiana degli anni ' 90 del secolo precedente, ovvero il periodo in cui è ambientato il romanzo, e mettono in rilievo proprio i pregiudizi che si dovrebbero evitare per rifiutare una società che non garantisce l'equità sociale nonché per poter fare il passo verso una società giusta, definita dagli ecocritici la csd. «società orizzontale», nella quale le differenze culturali, religiose, etniche, ecc. vengono percepite ed utilizzate per arricchire tutti i membri della società.

Tuttavia, nonostante il personaggio cercasse di dimostrare di essere una brava persona, che si trova in Italia non per rubare ma per cercare un lavoro e per migliorare le condizioni della propria vita, questi tentativi risultano vani perché i pregiudizi sono talmente presenti nell'immaginario collettivo delle persone che il protagonista non può fare niente per evitare di essere definito secondo i pregiudizi e gli stereotipi che riguardano il suo paese d'origine, e, a volte, anche altri paesi: "Mi piacciono le sue tette. Lei intanto dice che i marocchini sono cattivi perché, per un niente, tirano fuori il coltello, 
ma gli indiani no: sono bravi." (Methnani - Fortunato 2006:33) Questi esempi sono così numerosi nell'opera e mettono in rilievo una «topografia etnica», ovvero le descrizioni di zone delimitate dalla presenza quasi esclusiva di singole etnie, in modo tale che in una città vi si possono scoprire le zone circoscritte dalle caratteristiche particulari che delineano questi gruppi etnici, per rintracciare, infine, le vere e proprie «frontiere» all'interno di una città:

A poco a poco, ho imparato a riconoscere i luoghi di appuntamento, secondo le etnie e le provenienze. Roma ha una mappa alternativa a quella che trovi allegata alle Pagine gialle. Per esempio, uno vuol sapere dove si incontrano i libici? Semplice: in un bar di via Gioberti, un bar che fa angolo. I senegalesi invece preferiscono incontrarsi nei giardini di Colle Oppio, e i filippini in piazza Risorgimento [... . Mentre noi tunisini ci muoviamo fra i bar di piazza Esedra e quelli di piazza dei Cinquecento. $\dot{E}$ un'autentica topografia di secondo livello, questa, una sorta di circuito underground alla luce del sole, con le sue regole, e i suoi confini ben definiti. (Methnani - Fortunato 2006:57)

Perciò Methnani accenna all'incomunicabilità che spesso caratterizza i paesi con un alto numero di immigrati e le conseguenze di essa:

L'esistenza di incomunicabilità in una società come quella italiana, dove convivono fianco a fianco da alcuni anni una popolazione autoctona maggioritaria detentrice di una cultura locale, e una popolazione minoritaria composta di nuovi arrivati portatori di tradizioni differenti porta inevitabilmente alla manifestazione di tensioni identitarie. Tensioni che sono determinate nella maggior parte dei casi, come ricorda Maalouf da due concezioni estreme vis-à-vis della immigrazione e degli immigrati: "una che vede il paese di accoglienza come una pagina bianca su cui ciascuno potrebbe scrivere ciò che gli piace. Un'altra che vede il paese di accoglienza come una terra le cui leggi, i cui valori, le cui caratteristiche culturali e umane sarebbero già fissati una volta per tutte, e a cui gli immigrati non dovrebbero far altro che conformarsi". Due visioni ugualmente prive di realismo, sterili e nocive. Una via di mezzo andrebbe per forza ricercata. Ma da chi? (Methnani 2008:2)

Le domande poste da Methnani sono due: in primo luogo, come evitare di percepire il paese di accoglienza in uno dei due modi, o come un paese in cui «è lecito» fare quasialsi cosa o come il paese in cui le leggi sono già definite e creano un dogma al quale non si può che obbedire e, poi, chi, soprattutto, dovrebbe cercare la via di mezzo tra le due visioni estreme della società in cui si immigra; la risposta a tutte e due le domande la troviamo proprio nelle opere degli autori immigranti della csd. seconda generazione, ovvero quelli che sono, se non nati, allora almeno cresciuti in Italia e I'hanno vissuta per tanto tempo come una seconda patria. Saranno loro che non avranno bisogno di, per avere delle conferme sulla propria identità, emigrare. Anzì, nel caso di questi autori, il viaggio sarà in direzione opposta, verso il paese d'origine.

\title{
3. Conflitto Con il Paese D'origine
}

Daniela Golfetto, studiosa della scrittura migrante e della scrittura creativa, mette in rilievo alcuni aspetti fondamentali per la scrittura migrante femminile in Italia:

\begin{abstract}
La produzione femminile è un tratto caratteristico della letteratura migrante, che fin dall'inizio si avvale di una massiccia presenza di scrittrici; [...]. Le autrici rappresentano oggi circa la metà della produzione totale della letteratura migrante, percentuale molto maggiore rispetto alla loro presenza nella letteratura italiana contemporanea. L'elevato numero di donne è dovuto a varie ragioni, prima fra tutte il fatto di appartenere ad una letteratura che si situa fuori dai dettami dall'industria culturale. (Golfetto 2010:1)
\end{abstract}

Quello che accomuna le scrittrici della letteratura migrante è "un percorso di liberazione e di nuova consapevolezza della propria diversità e della propria ricchezza, che accomuna queste autrici e che le spinge a scrivere in un'altra lingua." (Golfetto 2010:2) Quindi, da una parte, queste scrittrici si liberano di alcuni vincoli che la loro cultura di origine pone alle donne, mentre, dall'altra, si trovano davanti la possibilità di costruirsi una nuova identità e di tentare di viverla proprio come se fosse quella acquisita con la nascita. Spesso in questo percorso di formazione e di ricostruzione succede che queste scrittrici "si interrogano più delle altre sulla propria identità sospesa fra due culture, e sul rapporto conflittuale con il paese d'origine, come fanno ad esempio le indiane Gabriella Kuruvilla e Laila Wadia." (Golfetto 2010: 3) Bisogna, comunque, tenere a mente che, sia per il fatto che in questo caso parliamo di scrittrici e non di scrittori, sia per il fatto che parliamo di una seconda generazione di scrittori immigranti rispetto a quella di Salah Methnani, sarà possibile notare varie differenze nelle loro opere, forse, in primo luogo, perché le scrittrici immigranti espatriano dai paesi che pongono diversi vincoli alla loro vita, nei quali non si potevano esprimere liberamente e si trovano in una cultura che è meno patriarcale rispetto alla loro cultura d'origine, il che permette loro di costruire quasi «una seconda identità». 
Tuttavia, anche nel paese di accoglienza devono scontrarsi con una cultura maschilista, e, di conseguenza, devono premunirsi contro il rischio di, alla fine, non riconoscersi in nessuna delle due identità. II loro percorso è, quindi, doppiamente marginalizzante, sia essendo donne sia essendo immigranti. Ciò significa che la loro scrittura ne sarà doppiamente affetta e che il loro percorso sarà ancora più sofferente e più maturo rispetto a quello della prima fase degli scrittori immigranti. Quello che caratterizza fortemente la loro scrittura è che, da un lato, nella nuova società troveranno numerosi casi di maschilismo e di pregiudizi nei confronti delle donne straniere, mentre, dall'altro, proprio a causa di un percorso diverso rispetto agli scrittori immigranti, osserveranno la nuova società con un altro sguardo, più consapevoli che le differenze non sono nient'altro che «le armi» che la gerarchia tradizionalmente imposta nella società «verticale» usa per tenere sotto controllo i membri «deboli».

La raccolta di racconti Amori bicolori, tra i quali troviamo anche il racconto Nascita di Ingy Mubiayi, è stata pubblicata per la prima volta nel 2008. In questo racconto la scrittrice non problematizza il rapporto dei protagonisti con il paese di accoglienza, dato che, essendo cresciuti in un altro paese, questi protagonisti sono coscienti dell'importanza di integrazione e della convivenza pacifica nel nuovo paese e riescono ad integrarvisi. Questa maturazione, tuttavia, ha anche le conseguenze negative e li porta spesso verso la diffidenza nei confronti del paese d'origine, il quale nella loro mente si trasforma in un concetto lontano, che incuriosice, ma, allo stesso tempo, spaventa e fa allontanare.

II ritorno nel paese d'origine coincide con l'atto della nascita della figlia della protagonista; nel momento in cui diventa madre, la protagonista comincia a domandarsi sul proprio rapporto con il paese d'origine, ovvero inizia a cercare le radici, ripensando alla propria nascita, ora che ha messo al mondo una figlia. La nascita a cui si allude nel titolo è, quindi, una doppia nascita, sia della figlia sia della madre, siccome tutte e due si trovano nel momento quando bisogna porre le basi per la propria vita e capire l'importanza della propria origine. Perciò all'inizio del racconto la protagonista rileva: "Mia figlia. Non riesco ancora a impadronirmi totalmente di questo concetto. Come tutte, mi sento catapultata in un'altra dimensione. [...] Eppure c'è qualcosa che mi sfugge, a cui io sono sfuggita per mesi, per questi anni." (Mubiayi 2013:48) L'incapacità di far parte di una famiglia o di un insieme di individui legati da certi vincoli, che la nascita della bambina causa nella protagonista, è legata soprattutto al fatto che, nell'affrontare l'importanza della provenienza ed appartenenza ad un gruppo etnico, si è trovata smarrita e senza radici ("Era vero, eravamo straniere in terra straniera, ma sua figlia sarebbe stata straniera anche tornando indietro. Così tanto valeva provarci." (Mubiayi 2013:57)). Risulta che, ora che è diventata responsabile per un altro essere umano, questo bisogno di conoscere e capire il proprio paese d'origine e la sua cultura si trasforma in lei in un'esigenza che non può più essere rimandata. Più precisamente, il distacco dal corpo materno della bambina riporta la protagonista a farsi le domande sul proprio distacco dalla madre e, contemporaneamente, sul rapporto con la patria. Riguardo a ciò, ricordiamo le parole di Lea Melandri che ci ricorda che "[...] la storia appare attraversata da un movimento opposto: corsa verso il passato, «regressione fin dentro il ventre materno». Nostalgia e speranza coincidono: il paradiso a cui si mira è lo stesso da cui siamo partiti." (Melandri 2001:21) Questa idea di ritorno, che continuamente segna la vita umana, secondo la Melandri, è legata ad un dato biologico, grazie al quale l'uomo è in continuazione ossessionato dal bisogno di doversi ricongiungere con la madre, ma anche "di non essersene mai staccati del tutto" (Melandri 2001:21). Perciò l'uomo si trova in continuazione sperduto tra il bisogno di sentire il legame attraverso il cordone ombelicale e la necessità di staccarsene. Lo stesso succede nell'uomo per quanto riguarda il suo rapporto con il paese di origine: non si riesce mai a lasciarselo indietro, e si continua a sentire le proprie radici per tutta la vita, nonostante ci si sia trasferito in un altro paese. Perciò non sorprende che proprio ora che è diventata madre, la protagonista sente un forte bisogno di conoscere la patria e ritrovare il padre di cui non ha molti ricordi. Quindi, il rapporto con la patria simboleggia, in questo caso, il rapporto tra la figlia e il padre, ed è quello con il quale la protagonista deve fare i conti prima di poter affrontare tranquillamente il futuro. II concetto dello straniero in questo racconto appare conseguentemente del tutto diverso dallo straniero di cui si parla nell'opera di Methnani, perché il sentimento di mancanza delle radici e di estraneità nascono nella protagonista, non come conseguenza di un viaggio, dell'espatrio, ma proprio il motivo per farlo.

Di conseguenza, appare chiara la decisione della protagonista di fare un viaggio nel paese d'origine, portandovi anche la figlia, nonostante nemmeno lei stessa sia del tutto convinta che bisogni affrontare questo viaggio: "A che pro andare? Cosa penso di risolvere? Cosa penso che cambierà nella mia vita?" (Mubiayi 2013:49) e nonostante abbia programmato il rientro dopo sole quattro ore ("Basteranno quattro ore per giungere alla verità? Basteranno per rappacificare quell'altra me che destatasi sputa tutto il suo rancore?" (Mubiayi 2013:57)). Tuttavia, la protagonista affronta questo viaggio nonostante l'atteggiamento contrario di entrambe le famiglie, per esaudire il desiderio di ritrovare il padre che non vede da quando era piccola ("Mia madre non ne capiva il senso. A che pro? mi chiedeva come me lo chiedo io ora. Adesso hai una famiglia, su quella ti devi concentrare. II passato è andato, niente e nessuno te lo potrà ridare." (Mubiayi 2013:50)) Ma il passato non se n'è andato per la protagonista e rimane una questione da affrontare per poter 
continuare a vivere. ${ }^{1}$ Se avesse potuto lasciarlo sepolto, significherebbe che il passato le garantiva la tranquillità e la serenità, ma il silenzio nei confronti e dalla parte della figura paterna avevano aperto un vuoto incolmabile nella protagonista, il quale si poteva riempire soltanto affrontando il padre. Questo viaggio si rivela, quindi, un viaggio nel passato, ovvero il viaggio per trovare le risposte alle domande sulla propria identità, sia per quanto riguarda la questione etnica, sia per quanto riguarda il rapporto tra la figlia e il padre ("Ora che avevo quell'indirizzo non potevo più cambiare strada, dovevo affrontare quel mondo che pur non appartenendomi era mio" (Mubiayi 2013:51)). II padre era straniero nel paese di origine della madre, e ciò significa che già nel passato questa famiglia ha conosciuto la condizione dello straniero. Nella figura del padre, quindi, la protagonista cerca di capire l'importanza delle radici per una persona che si trova lontano dal proprio paese d'origine, e lo fa per capire se stessa e per trovare nelle azioni che il padre ha compiuto le risposte a certi propri atteggiamenti. Da ciò possiamo capire che questo tipo di scrittura migrante, che riguarda la seconda generazione di scrittori di letteratura dell'immigrazione, non si concentra sulla nuova vita dell'immigrante nel paese di accoglienza, ovvero sulla csd. «seconda patria», ma vi viene focalizzato il suo paese di origine. Quindi, nella seconda generazione degli autori immigranti il problema non consiste nell'affrontare la nuova società ed integrarvisi, ma nell'affrontare il paese di origine, di cui tanti aspetti rimangono, dopo gli anni di vita nel paese in cui si sono trasferiti, ancora segreti.

La madre, in questo racconto, simboleggia la nuova patria, il mondo in cui i nuovi residenti si sono del tutto integrati, e i quali si sono lasciati alle spalle un passato doloroso e non ne vogliono più sapere niente, nonostante la società nella quale si sono trasferiti non abbia del tutto soddisfatto le loro speranze:

Aveva lasciato il suo mondo classista e razzista, ne aveva trovato uno ancor più feroce che non sapeva cosa farsene del suo diploma, [...]. La sognatrice era stata sotterrata, la caparbia diventava più tenace che mai. La società più giusta che si aspettava non era mai arrivata. Si trasformavano invece le disparità. Non più la provenienza di ceto contava ma quella geografica. Non più differenza tra Nord e Sud del paese, ma del mondo. (Mubiayi 2013:57-58)

La maternità ha un ruolo importante anche quando riguarda la figlia: com'è stato già rilevato, ora che lei è diventata madre, tocca a lei riscoprire la seconda patria, costruendo, allo stesso tempo, l'equilibrio con il paese d'origine.

Alcuni elementi, però, il romanzo di Methnani e il racconto di Mubiayi hanno in comune: come nel romanzo di Methnani si "racconta di un egiziano che ha una paninoteca in Via Palazzuolo e che, nel suo locale, non fa entrare gli arabi" (Methnani - Fortunato 2006:76) e il quale, di conseguenza, viene definito razzista, così anche in questo racconto il razzismo e la discriminazione non sono associati soltanto agli italiani 0 al paese di accoglienza, ma a tutte le comunità, dato in qualsiasi «società verticale» non si possono evitare i dualismi e le discriminazioni. Tenendo ciò a mente, si capisce perché per la madre, con il viaggio che la figlia sta per intraprendere, vengono alla superficie tutte le paure sulle scelte, giuste o sbagliate, e sulle decisioni fatte tempo fa, nel momento in cui aveva deciso di cambiare vita andandosene via dal luogo in cui era nata:

Temeva di aver sbagliato. Ma temeva anche che nessun senso di colpa lo rodeva e se anche avesse provato un po' di rimorso, il nostro incontro lo avrebbe fatto morire in pace, nel perdono. [...] Perché mi sia accanita su quest'idea non l'ho capito ancora. Forse per lei, forse per me. Ma affrontare il senso di colpa che abbatte l'animo quando si provoca sofferenza alla propria madre è stata una delle prove più difficili da superare. (Mubiayi 2013:51)

Accanto alla protagonista e a sua madre, nella storia viene descritto un altro protagonista, il fidanzato, il cui personaggio simboleggia sia il paese in cui si è trasferita la protagonista, ovvero la sua «seconda» patria, sia la figura paterna con la quale la protagonista ha ancora un conto in sospeso. Questo aspetto diventa ancora più importante per il rapporto dei due fidanzati se si tiene a mente che il padre viene etichettato come «uno straniero» dalla parte della protagonista ed è questa estraneità lo stato che la protagonista vuole evitare:

Pur essendo io la straniera qui, pur vivendo nel suo di paese, ai miei occhi e agli occhi di quella comunità alla quale nemmeno io appartengo lui è uno straniero. Camminare sospesi tra due mondi può essere divertente, ma non facile, [...]. Non sapere a chi si appartiene, a chi rimettere il fulcro della propria identità mette in imbarazzo lo spirito. (Mubiayi 2013:52)

\footnotetext{
1 Proprio volendo riuscire ad avere $i$ rapporti equilibrati con entrambi i genitori, la protagonista è cosciente della necessità di affrontare $i$ punti dolenti nel rapporto con il padre. A proposito, parlando dell'amore dei genitori, e in particolare dell'amore materno, Marilde Trinchero ricorda che spesso ci si trova, involontariamente, in una specie di labirinto dal quale nessuna delle due, né la madre né la figlia, escono (Cfr. Trinchero 2008:95).
} 
Di conseguenza, osservando il rapporto del fidanzato con la figlia dei due, e cercando di trovarvi le risposte per il proprio rapporto con il padre, la protagonista nota la differenza tra un uomo e una donna nel rapporto con i figli, il che causa allontanamento e diffidenza in lei nei confronti del maschio: "Lui spinge la carrozzina come fosse un mezzo su cui viaggia qualcosa di prezioso ma con cui ha confidenza, non come un suo prolungamento. Sarà forse questo il segreto per renderli autonomi? o è l'attavica differenza nel rapporto tra padre e madre?" (Mubiayi 2013:49). In più, il rapporto con il fidanzato porta alla superficie un altro problema: questo rapporto si mostra, in questa catena di rapporti pieni di questioni irrisolte, anch'esso un anello complicato; a questo punto salta fuori la differenza tra i due, la differenza nella provenienza e nell'appartenenza ad un altro gruppo etnico, che alla protagonista appare come una voragine che non si può colmare, e che le persone, osservandoli, notano:

"Famiglia mista», suona strano, meglio "coppia mista con bambino». Lei scura, lui chiaro, e il bambino il giusto compromesso. Silenziosi, penseranno. Infelici, forse. Come se il silenzio fosse indice di mancanza. Chissà se funziona, si chiederanno. Chissà se funziona, me lo chiedo anch'io ora. Io nera, lui bianco. Famiglie d'origine distanti luce. [...] Le differenze ci sono e non basta l'istruzione o il denaro per colmarle. (Mubiayi 2013:49)

I pregiudizi che nota negli occhi delle persone intorno fanno scatenare in lei un dubbio: l'incertezza che una coppia con origini differenti ed etnicamente diversa, possa vivere una vita felice, anche perché altre persone intorno a lei non ne sono convinti: "Poi il prolungamento della nostra storia aveva destato segni di curiosità mista a sorpresa. Per parenti e amici io ero una straniera. Non uguale l'educazione, non uguali i valori. Orizzonti diversi, limiti geografici e morali diversi." (Mubiayi 2013:53) L'Italia si rivela, nonostante le aspettative diverse, un paese in cui continua a predominare un atteggiamento classista e razzista: "Non eravamo poi così diversi io e lui, ma lo eravamo agli occhi degli altri, lo eravamo rispetto agli altri." (Mubiayi 2013:54) Quindi, anche in questo racconto, come nel romanzo di Methnani, i pregiudizi e le gerarchie sono frequentemente presenti e forzano i protagonisti a chiedersi sulla possibilità di poter convivere con i pregiudizi o no. "Provate a volare come le foglie degli alberi, ad allontanarvi da quel pezzetto di terra che vi ha generati. Provate a nutrire e a nutrirvi d'altro", raccomanda ai lettori la voce narrante, ricordando la storia della madre, che ha lasciato il proprio paese. A questo punto scopriamo la differenza tra i due testi: nel racconto di Mubiayi, non ci si sofferma a subire passivamente le conseguenze dell'emarginazione e discriminazione, ma sono proprio gli immigrati coloro che cercano di vincere i pregiudizi e di cambiare la società che hanno accettato come la loro patria: "L'altro siamo noi. Siamo uguali, siamo uomini e donne che vogliono amare e vivere in pace." (Mubiayi 2013:52) Alla fine del suo viaggio, la protagonista capisce che la vera differenza stava tra la madre e il padre in quanto incompatibili di carattere, non perché di origine diversa. Risulta che, se si vuole costruire una società equa, si deve tenere a mente questo aspetto, cioè il fatto che la differenza tra le persone deve essere sfruttata per migliorare le condizioni di vita di tutti i membri di una società e non per dividerli. Se no, il rischio che si corre è che, con la società che presuppone la gerarchia ed il dualismo, le vittime sono inevitabili, e queste vittime sono in primo luogo i figli degli immigrati: " $E$ poi sono arrivata io. La sintesi, il ponte che avrebbe unito definitivamente due sponde. [...] Non hanno pensato che quella creatura avrebbe portato con sé non metà dell'uno o metà dell'altro ma interi continenti su di sé e in sé." (Mubiayi 2013:54)

Quindi, la vera svolta nella percezione della realtà e della società contemporanea la danno gli immigrati, i quali capiscono che "ii] giovani sono giovani ovunque, i vecchi fanno i vecchi ovunque." (Mubiayi 2013:52) Nel caso della protagonista, si scopre alla fine che "[l]'uomo che veniva da oltre il deserto aveva mentito. Non sono stati né religione né usanze diverse a spezzare l'incantesimo, ma la banalità dell'inganno. [...] L'uomo nero aveva semplicemente un'altra famiglia [...]."(Mubiayi 2013:58) Questo dato conferma che le differenze geografiche, legate alla provenienza e all'appartenenza a diversi gruppi etnici, non contano più nella società contemporanea, e devono essere superate. Concludiamo che in questo racconto «l'altro», alla fine, scompare, come scompare la differenza in base all'appartenenza etnica 0 qualsiasi altra caratteristica che differenzia le persone. Ciò che rimane è un semplice uomo, che fa le scelte e compie gli atti secondo la propria coscienza, ma non in quanto differente per origine, per colore della pelle, ecc. Quando la protagonista lo capisce, può finalmente lasciarsi alle spalle il passato e mettere la parola fine alle questioni riguardo alla figura paterna e, soprattutto, riconquistare l'equilibrio con il paese d'origine.

\section{Conclusione}

Nel romanzo di Methnani seguiamo la trasformazione di un personaggio arrivato in Italia nella ricerca di un lavoro e con la speranza di una vita migliore, ad un uomo smarrito e disilluso. Però, allo stesso tempo, parallelamente con il peggioramento delle condizioni di vita che il trasferimento gli ha portato, si ha anche la crisi dell'identità alla quale subentra l'indifferenza e rassegnazione: 
A cercare un lavoro, ho rinunciato del tutto. [...] Tutto è diventato normale e indifferente. Scivolo lungo una strada che non conduce da nessuna parte, ma non importa. [...] Mi sento un palloncino che vola di qua e di là. [...] Anch'io sono un tossico, adesso. Un tossico clandestino. Finalmente, ho un'identità. [...] Tutti quelli che incontro mi sembrano simili a me. Niente differenze, nessuna esclusione. (Methnani - Fortunato 2006:74-80)

Si può constatare che, in questo romanzo, la ricerca dell'identità scatta dopo il momento della migrazione, nella società nuova e diversa da quella del paese d'origine. Dopo l'iniziale smarrimento, il protagonista si trova a cercare di capire chi è veramente, e, nell'incontro con altri, che lo etichettano come diverso da loro e, di conseguenza, lo emarginano, l'unica via d'uscita è avvicinarsi ad altri immigrati, gli unici nei quali riesce a vedere le somiglianze e a placare temporaneamente lo stato d'animo. In questo modo, essendo solo «un immigrato», trova il modo per vivere non rilevando le differenze che questa «società verticale» gli fa continuamente notare dal momento che ci è arrivato. Nell'opera di Ingy Mubiayi, invece, la ricerca dell'identità scatta nel momento in cui la condizione del protagonista cambia, ma non cambia il paese in cui risiede; in quel momento lei si vede costretta a farsi le domande e a cercare le risposte sulla propria identità, le quali può trovare soltanto tornando nel paese d'origine.

Partendo dal pressupposto che "la letteratura, e la cultura in generale, sono un modo attraverso il quale l'essere umano rappresenta i propri valori e il proprio rapporto con il mondo che lo circonda" (lovino 2006:62), gli esempi della letteratura d'immigrazione in Italia offrono la possibilità di individuare, nella società italiana degli ultimi decenni, l'assenza di pari opportunità e dell'equità sociale e, allo stesso tempo, lo sforzo di riempire queste mancanze da parte di alcuni membri della società. In tutti e due i testi troviamo gli esempi di pregiudizi ed i tentativi di emarginazione, i quali rivelano le cattive abitudini di alcuni membri della società italiana nei confronti degli immigrati. Tuttavia, dato che le due opere sono state pubblicate a distanza di diciotto anni, è possibile notare alcuni cambiamenti nella letteratura d'immigrazione in Italia: mentre nelle prime opere sono stati focalizzati gli immigranti stessi e vi venivano raccontate le condizioni di vita in cui gli immigrati erano costretti a cercare di sopravvivere, rivelando una realtà oscura e vergognosa, nella seconda generazione degli scrittori immigranti la realtà che questi vivono è parecchio cambiata, anche perché questi scrittori vivono in Italia dall'infanzia e la vivono come una seconda patria. "La clandestinità non fa parte della mia esperienza e quei quattro amici stranieri che ho, li ho incontrati strada facendo e non sono nemmeno delle mie zone" (Mubiayi 2013:524), rileva la voce narrante del racconto di Ingy Mubiayi. La scrittrice racconta una società diversa, un mondo in cui l'immigrato non è più soltanto uno straniero 0 un immigrato, come nel caso del romanzo di Methnani, e si sente più straniero nel paese di origine del proprio genitore ("[... gli uomini e le donne di al di là del deserto mi sentiranno sempre diversa e mai mi accetteranno perché il colore della pella non basta a farti parte di una comunità" (Mubiayi 2013:68)). Questo significa che nelle opere degli autori immigranti di seconda generazione, ai quali appartiene anche Ingy Mubiayi, sarà possibile percepire il tentativo dell'integrazione nel paese in cui si è immigrati e il risultato di questo processo; anzì, nell'atteggiamento dei protagonisti si potrà notare il rapporto conflittuale con il paese d'origine, conosciuto magari soltanto attraverso il racconto dei genitori e non di persona. Le scrittrici migranti di seconda generazione, come, ad esempio, Igiaba Scego e Laila Wadia, offrono nelle loro opere numerosi esempi del genere, dei casi in cui i protagonisti si trovano a dover combattere contro i parenti per farli convincere di accettare la nuova cultura, quella italiana, in quanto quella che hanno lasciato non è più l'unica che li caratterizza, e loro insistono nel trovare il modo per far convivere le «due identità», le due culture, in equilibrio, senza danneggiare nessuna delle due. Questa è, spesso, una lotta tutta al femminile, dove le donne vogliono non solo fare spazio alla realizzazione della parità dei diritti femminili nella società maschilista, ma vogliono soprattutto creare le basi per una società più equa, la quale rappresenta l'unica possibilità per uscire dalla crisi contemporanea. Questa lotta per loro rappresenta sia la libertà dai pregiudizi nei confronti del gruppo etnico al quale appartengono, ma è anche una lotta per i diritti della donna, la quale si trova, nel paese di accoglienza, a dover combattere contro i pregiudizi e la discriminazione nei confronti delle donne e di tutti i membri «deboli». Perciò questo tipo di scrittura non solo denuncia la presenza della cosiddetta «società verticale» alla quale fanno riferimento frequentemente gli ecocritici, ma offre anche gli esempi come uscirne, ovvero come superare le differenze ed accettarle come il punto forte di tutti i membri di una comunità e non percepirle come le basi per promuovere la discriminazione. Dall'analisi risulta che la letteratura migrante offre vari esempi di come poter porre, nella società contemporanea, le basi per una cultura più giusta che avrebbe, come conseguenza, un modo di vita sostenibile.

\section{References}

Buell, L. (2005). The Future of Environmental Criticism. Environmental Crisis and Literary Imagination, Malden\&Oxford\&Victoria: Blackwell Publishing

Garrad, G. (2004). Ecocriticism. London-New York: Routledge.

Glotfelty, C. (1996). Ecocriticism: literary studies in an age of environmental crisis. In Di Giulio, R. T. et al. (eds.) Interconnections 
between Human and Ecosystem Health (pp. 229-236). London: Chapman\&Hall.

Iovino, S. (2006). Ecologia letteraria. Una strategia di sopravvivenza. Milano: Edizioni Ambiente.

Kuruvilla, G. - Mubiayi, I. - Scego, I. - Wadia, L. (2013). Pecore nere, e-book, Roma - Bari: Gius. Laterza \& Figli.

Melandri, L. (2001). Le passioni del corpo. La vicenda dei sessi tra origine e storia. Torino: Bollati Boringhieri.

Methnani, S. - Fortunato, M. (2006). Immigrato. Milano: Tascabili Bompiani.

Mihaljević, N. (2012). Some ecological issues in a few Luigi Pirandello's short stories. In "Mediterranean Journal of Social Sciences", vol. 3 , No. 10, Rome, pp. 285-291;

Mubiayi, I. (2013). Nascita. In Masri, M,. - Mubiayi, I. - Qifeng, Z. - Scego, I. Amori bicolori, e-book, Roma-Bari: Gius. Laterza \& Figli, pp. 48-69.

Trinchero, M. (2008). La solitudine delle madri. Roma: Edizioni Magi.

Ellero, P. Letteratura migrante in Italia. In Lingua Nostra, e Oltre, Anno 3, Numero 3 - 5, (http://www.maldura.unipd.it/masters/italianoL2/ Lingua_nostra_e_oltre/LNO3_26luglio2010/Ellero_4_12.pdf) (25.03.2014.), pp. 4-12.

Golfetto, D. Scrittura migrante, scrittura creativa. La voce femminile della letteratura migrante in Italia. (estratto, senza adattamenti formali, dalla tesina di fine Master (a.a. 2008-2009) In Lingua Nostra, e Oltre, Anno 3, Numero 3-69, (http://www.maldura.unipd.it/masters/italianoL2/Lingua_nostra_e_oltre/LNO3_26luglio2010/Gofetto_69_71.pdf) (02.04.2014.), pp. 1-3.

Luzi. A. (2008). Migrazione e identità: Immigrato di Salah Methnani. (http:// http://www.disp.let.uniroma1.it/kuma/intercultura /kuma15luzi.pdf) (11.04.2014.), pp. 1-10.

Methnani, S. (2008). Sguardo italiano e identità dell'altro. (http:// http://www.disp.let.uniroma1.it/kuma/intercultura/kuma15methnani.pdf) (13.04.2014.), pp.1-3. 